\title{
Planning, Managing and Monitoring Technological Security Infrastructures
}

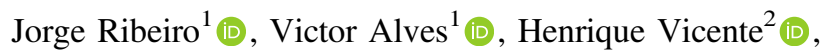 \\ and José Neves ${ }^{3(\mathbb{})}(\mathbb{D})$ \\ 1 ARC4DigiT - Applied Research Center for Digital Transformation, \\ School of Technology and Management, \\ Polytechnic Institute of Viana do Castelo, Viana do Castelo, Portugal \\ jribeiro@estg.ipvc.pt, victoralves.at.vnc@gmail.com \\ 2 Department of Chemistry, School of Sciences and Technology, \\ Évora Chemistry Centre, University of Évora, Évora, Portugal \\ hvicente@uevora.pt \\ ${ }^{3}$ Department of Informatics, School of Engineering, ALGORITMI Centre, \\ University of Minho, Braga, Portugal \\ jneves@di.uminho.pt
}

\begin{abstract}
Over the past few decades many different Information Technologies (IT) policies have been introduced, including COSO, ITIL, PMBook, CMM, ISO 2700x, Six Sigma, being COBIT IT (Control Objectives for IT) the framework that encompasses all IT and Information Systems (IS) governance activities at the organization's level. As part of the applicability of quality services certification (ISO 9001) in all IT services of a public institution, it is presented a case study aimed at planning, managing and monitoring technological security infrastructures. It followed the guidelines for the ISO $2700 \mathrm{x}$ family, COBIT, ITIL and other standards and conducted a survey to complement the IT process's objectives. With regard to an action-research methodology for problem-solving (i.e., a kind of attempt to improve or investigate practice) and according to the issue under analyze, the question is put into the terms, viz. "How can the ISO 2700x, COBIT, ITIL and other guidelines help with the planning, management and monitoring of technological security infrastructures and minimize the risk management of IT and IS?'. Indeed, it may be resolved that it is possible to achieve the goals of planning, managing and monitoring a technological security infrastructure. In the future, we will use Artificial Intelligence based approaches to problem solving such as Artificial Neural Networks and Cased Based Reasoning, to evaluate this issue.
\end{abstract}

Keywords: Artificial intelligence $\cdot$ Artificial neural networks Case based reasoning - Security infrastructures $\cdot$ COBIT $\cdot$ ITIL ISO $27001 \cdot$ ISO 27005 